Research Paper

\title{
Sesamin inhibits cervical cancer cell proliferation by promoting p53/PTEN-mediated apoptosis
}

\author{
Tian-Ni Kuo1, Chun-Shiang Lin², Guan-De Li³, Cheng-Yi Kuo ${ }^{4}$, Shao-Hsuan Kao, ${ }^{\natural}$ \\ 1. Department of Obstetrics and Gynecology, Chi Mei Medical Center, Tainan 710, Taiwan. \\ 2. Precision Medicine Center, Chung Shan Medical University Hospital, Taichung 402, Taiwan. \\ 3. Department of Medicine, Medical College, Chung Shan Medical University, Taichung 402, Taiwan. \\ 4. Department of Biology and Anatomy, National Medical Center, Taipei 114, Taiwan. \\ 5. Institute of Medicine, Medical College, Chung Shan Medical University, Taichung 402, Taiwan \\ 6. Clinical Laboratory, Chung Shan Medical University Hospital, Taichung 402, Taiwan
}

$\square$ Corresponding author: Institute of Medicine, Medical College, Chung Shan Medical University, No.110, Sec. 1, Jianguo N. Rd., Taichung 402, Taiwan. E-mail: kaosh@csmu.edu.tw Tel: +886-4-24730022 ext. 11681.

(c) The author(s). This is an open access article distributed under the terms of the Creative Commons Attribution License (https://creativecommons.org/licenses/by/4.0/). See http://ivyspring.com/terms for full terms and conditions.

Received: 2020.06.01; Accepted: 2020.08.17; Published: 2020.08.25

\begin{abstract}
Background: Sesamin is a major bioactive compound in sesame seeds and has various biological properties, including anti-inflammatory and anticancer activities. Here, we explored whether sesamin activates $\mathrm{p} 53$, which is widely inhibited in cervical cancer cells, thereby inducing p53-mediated apoptosis.

Methods: Human HeLa and $\mathrm{SiHa}$ cervical cancer cells and normal Hs68 dermal cells were used as cell models. Cell proliferation, cell cycle distribution, and apoptosis were evaluated by the CCK- 8 assay and flow cytometry using PI/Annexin V staining, respectively. Protein expression and phosphorylation were determined using western blotting. The involvement of p53 in the apoptotic cascade was assessed by a specific inhibitor.

Results: Sesamin (75 and $150 \mu \mathrm{M})$ clearly inhibited $\mathrm{SiHa}$ and HeLa cell proliferation in a dose-dependent fashion, but did not affect the proliferation of $\mathrm{Hs} 68$ cells. Meanwhile, sesamin increased the sub-G 1 phase ratio and apoptosis, up to approximately $38.5 \%$ and $37.8 \%$, respectively. Furthermore, sesamin induced p53 phosphorylation at serine-46 and serine-15 and upregulated the levels of PUMA, Bax, and PTEN, while inhibiting AKT phosphorylation at serine-473. Inhibition of $p 53$ by pifithrin- $\alpha$ significantly reduced the levels of PUMA, Bax, and PTEN but restored AKT phosphorylation in SiHa cells exposed to sesamin. Pifithrin- $\alpha$ also reduced apoptosis and restored the proliferation of $\mathrm{HeLa}$ and $\mathrm{SiHa}$ cells exposed to sesamin.

Conclusions: These findings indicate that sesamin inhibits cervical cancer cell proliferation, and its mechanism may be attributed to the induction of p53/PTEN-mediated apoptosis. This suggests that sesamin might be useful as an adjuvant in promoting anti-cervical cancer treatments.
\end{abstract}

Key words: Sesamin, Cervical cancer cell, p53, PTEN, PUMA, Apoptosis.

\section{Introduction}

Cervical cancer is a worldwide life-threatening malignancy in women. The common use of the pap smear for uterine neoplasia screening promotes early diagnosis of uterine cancer and significantly lowers the mortality of patients with uterine cancer [1]. However, cervical cancer remains a life-threatening malignancy in women worldwide [2]. The death of patients with late-stage cervical cancer is primarily attributed to recurrence and distal metastasis to lymph nodes, lungs, and liver [3-5]. Accordingly, the combination of surgical resection, chemotherapy, and radiotherapy is widely used to treat cervical cancer and prevent its recurrence and distal metastasis, and such treatments clearly prolong the overall and progression-free survival of patients with cervical cancer $[6,7]$.

Although chemotherapy has obvious effects on malignant cancer cells, it also has inevitable 
cytotoxicity on normal cells and may result in chemoresistance. In the last few years, natural compounds with potential anticancer activity isolated from dietary plants and medicinal herbs have been increasingly and extensively investigated [8]. Sesamin is a sesame lignan with a sesamin content of only $0.5-1.2 \%$ in sesame seeds. Mounting evidence has shown that sesamin has various properties, including antioxidant and anticancer activities.

TP53 is an important tumor suppressor gene, and its various mutant forms are widely found in most human tumors. However, in cervical cancer, p53 may be inactivated by binding to viral proteins, rather than through TP53 mutations. Notably, TP53 mutations are rarely found in cervical cancer, but more than $90 \%$ of cervical cancers are infected with human papillomavirus (HPV) [9]. The HPV E6 protein interacts with cellular proteins, E6-AP and p53, and promotes p53 degradation through the ubiquitin-dependent proteolytic system, which may result in p53 dysfunction in HPV-infected cervical tissues [10]. Thus, it has been suggested that p53 activation may be a potential anticancer strategy for the treatment of cervical cancer. In this study, we aimed to explore the antiproliferative effects of sesamin on cervical cancer cells, with an emphasis on the p53-mediated apoptotic cascade. Cell proliferation and cell cycle distribution were determined using the CCK-8 assay and flow cytometry, respectively. Protein expression and phosphorylation were assessed using western blotting. The involvement of p53 in the proapoptotic cascade was evaluated using a specific inhibitor.

\section{Materials and Methods}

\section{Cell culture and reagents}

Human cervical cancer cell HeLa and SiHa and dermal fibroblast Hs60 were purchased from Bioresource and Collection and Research Center (Hsinchu 300, Taiwan) and maintained in the culture medium [Dulbecco's modified Eagle's medium (DMEM) containing $2 \mathrm{mM}$ L-glutamine, $1.5 \mathrm{~g} / \mathrm{L}$ sodium bicarbonate, $0.1 \mathrm{mM}$ non-essential amino acids, $1.0 \mathrm{mM}$ sodium pyruvate, $100 \mathrm{U} / \mathrm{mL}$ penicillin, $100 \mu \mathrm{g} / \mathrm{mL}$ streptomycin, and $10 \% \mathrm{v} / \mathrm{v}$ fetal bovine serum (FBS, HyClone, Thermo Fisher, Waltham, MA, USA)]. Cells were maintained grown in a humidified incubator with $5 \% \mathrm{CO}_{2}$. All the reagents without specific indication were purchased from Sigma-Aldrich (St Louis, MO, USA).

\section{Cell proliferation assay}

Cell viability was assessed by using a Cell Counting Kit-8 (CCK8, Sigma-Aldrich). Briefly, cells were cultured in 96-well plates at an initial density of $3 \times 10^{3}$ per well, and then incubated with the indicated concentration of sesamin for 24 or $48 \mathrm{~h}$. After the incubation, $10 \mu \mathrm{L}$ CCK-8 solution was added to each well, and the plate was incubated at $37^{\circ} \mathrm{C}$ for $4 \mathrm{~h}$, then the absorbance at $450 \mathrm{~nm}$ was measured using a microplate reader (Molecular Devices, Sunnyvale, CA, USA). The cell viability was presented as mean \pm standard deviation at percentage of control.

\section{Cell cycle distribution analysis}

Cells were maintained in the serum-free culture medium for $16 \mathrm{~h}$ prior to treatments, to synchronize cell cycle, then treated with sesamin at the indicated concentrations for $24 \mathrm{~h}$ in the culture medium. After the treatments, cells were detached by incubation of trypsin, fixed with $70 \%$ ethanol, washed with PBS, then reacted with propidium iodide (PI, $20 \mu \mathrm{g} / \mathrm{mL}$ ) for 30 minutes in dark. After the reaction, the cells were subjected to cell cycle distribution analysis using a flow cytometer (BD Biosciences, San Jose, CA, USA). Ten thousand cells were counted for cell cycle distribution quantitation using CellQuest Pro software (version 5.1, BD Biosciences).

\section{Apoptosis assessment by Annexin V/propidium iodide assay}

Cells were grown to approximate $80 \%$ confluence, treated with DMSO, sesamin, or pifithrin- $\alpha$ following sesamin for $24 \mathrm{~h}$, and the cells were collected for cell apoptosis assessment by using an annexin V-FITC apoptosis detection kit (APOAF, Sigma-Aldrich) according to the manufacturer's instructions. Briefly, cells were washed with cold PBS, and then reacted with Annexin V- FITC and PI (20 $\mu \mathrm{g} / \mathrm{mL}$ ) in the dark for $20 \mathrm{~min}$. Apoptotic cells were analyzed immediately by BD FACScan flow cytometer (BD Biosciences) with CellQuest pro 5.1. For each sample, the fluorescence of 10,000 cells was gated and counted. The degree of apoptosis was presented as a percentage of the annexin $\mathrm{V}$ positive and PI-negative (annexin V+/PI-) cells.

\section{Western blot}

After treated with sesamin at the indicated concentrations for $24 \mathrm{~h}$, the cells were washed with PBS, detached by trypsin incubation, collected, and then lysed with the RIPA buffer containing protease and phosphatase inhibitors for crude protein extraction. The crude proteins were separated using SDS-polyacrylamide gel electrophoresis, then transferred onto an Immobilon P PVDF membrane (Merck Millipore, Billerica, MA, USA). The membranes were blocked with $5 \%$ skimmed milk for $1 \mathrm{~h}$, and then incubated with primary antibodies 
against human p53, phosphorylated p53 at serine 15 [p-p53(S15)], p-p53(S46), PUMA, Bax, PTEN, p-AKT(S473), and $\beta$-actin at $4^{\circ} \mathrm{C}$ for $16 \mathrm{~h}$. After the incubation, the membrane was washed with PBST (PBS containing 0.5\% Tween-20), and then incubated with the peroxidase-conjugated secondary antibody (Santa Cruz Technology, Santa Cruz, CA, USA). Antibody complex was detected using chemiluminescence development reagent (GE Healthcare, London, UK), and the resulting chemiluminescent signals were acquired and quantitated using a Luminescent Image Analyzer LAS-4000 mini (GE Healthcare).

\section{Statistical analysis}

Quantitative data were presented as means \pm standard errors. Statistical analysis was performed using SigmaPlot (version 11, Systat Software Inc.). One-way analysis of variance (ANOVA) and an unpaired 2-tailed Student's $t$-test was used to determine the significance of differences. $P$ value less than 0.05 was considered as significant.

\section{Results}

\section{Sesamin reduces the proliferation of HeLa and $\mathrm{SiHa}$ cells, but not $\mathrm{Hs} 60$ cells}

The effects of sesamin on the proliferation of cervical cancer cells were first investigated. Our observations showed that 24 -h sesamin treatments (15 $-300 \mu \mathrm{M})$ dose-dependently reduced the proliferation of $\mathrm{HeLa}$ and $\mathrm{SiHa}$ cells, by up to $74.3 \pm 4.2 \%$ and $70.5 \pm 2.4 \%$ of the DMSO control, respectively (Figure
1A, upper panel, $P<0.001$ ). In addition, 48 -h sesamin treatments further inhibited the proliferation of HeLa cell and SiHa cell, up to $69.2 \pm 5.2 \%$ and $58.9 \pm 2.8 \%$ of DMSO control, respectively (Figure 1A, lower panel, $P<0.001)$. In addition, the effect of sesamin on the proliferation of normal Hs60 fibroblasts was also explored. As shown in Figure 1B, sesamin only mildly decreased the proliferation of Hs60 cells, to $91.3 \pm 6.2 \%$ of the control at $300 \mu \mathrm{M}$ ( $48 \mathrm{~h}$ treatment, $P=0.044$ as compared to control). Taken together, these observations indicate that sesamin clearly inhibits the proliferation of cervical cancer cells, but not of normal dermal fibroblasts.

\section{Sesamin induces sub-G1 phase accumulation and apoptosis of HeLa and SiHa cells}

To further explore the inhibited cell proliferation in response to sesamin, we next analyzed the effects of sesamin on cell cycle distribution. As shown in Figure 2A, $75 \mu \mathrm{M}$ sesamin treatment clearly increased G0/G1 ratios, from $66.3 \pm 2.7 \%$ to $78.4 \pm 3.1 \%$ (HeLa) and from $64.3 \pm 3.4 \%$ to $81.3 \pm 3.6 \%$ ( $\mathrm{SiHa}$ ), respectively $(P<0.05$ as compared to DMSO control). In addition, $75 \mu \mathrm{M}$ sesamin also increased sub-G1 ratios, to $8.3 \pm 1.9 \%$ and $7.8 \pm 2.3 \%$ in HeLa and SiHa cell, respectively $(P<0.01$ as compared to DMSO control). Interestingly, $150 \mu \mathrm{M}$ sesamin dramatically increased sub-G1 ratios, to $19.1 \pm 2.4 \%$ and $38.4 \pm 2.7 \%$ in HeLa and $\mathrm{SiHa}$ cells, respectively ( $P<0.005$ as compared to DMSO control), while decreasing G0/G1 ratios in both cell types. In addition, annexin V/PI staining was used to demonstrate whether sesamin induced cell apoptosis. As shown in Figure 2B, sesamin clearly increased
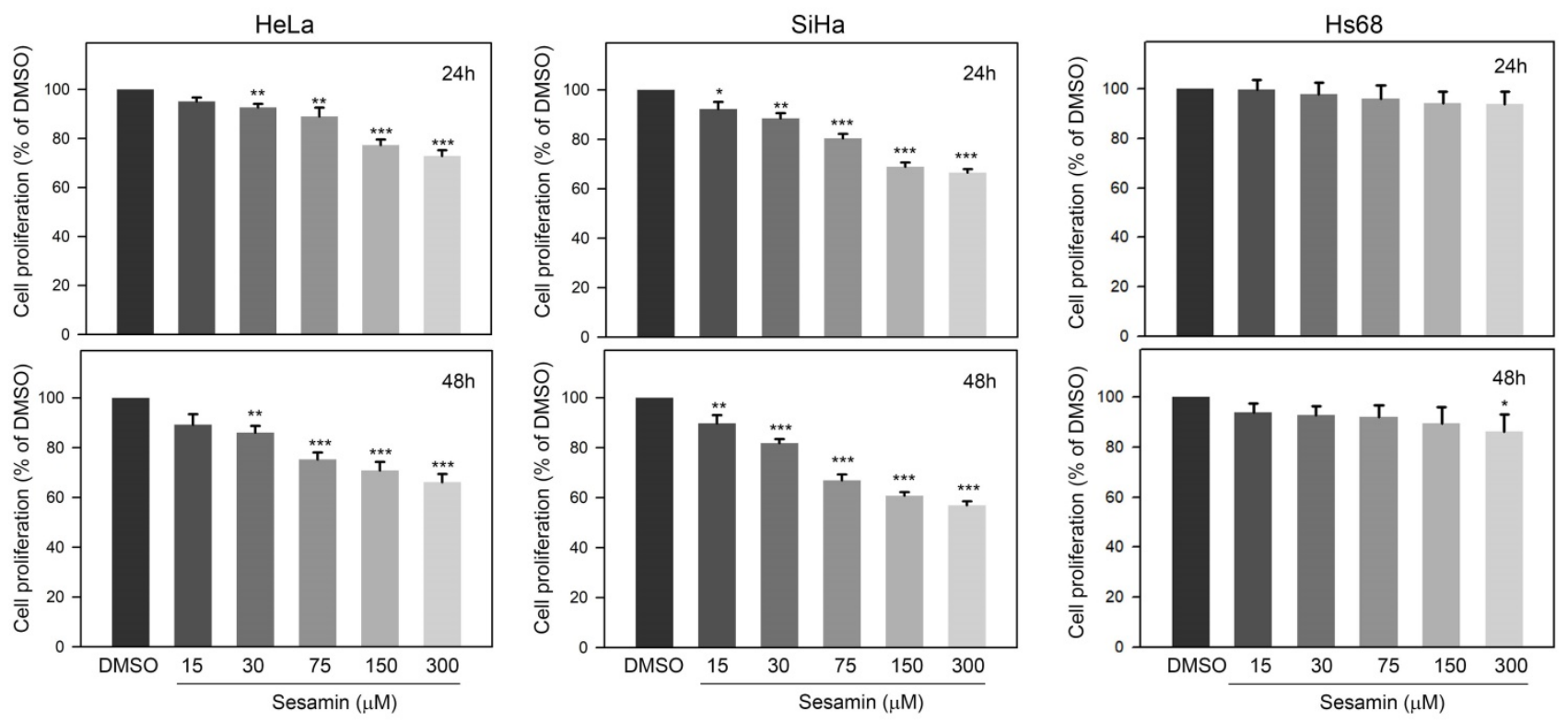

Figure 1. Effects of sesamin on cell proliferation of human cervical cancer cell HeLa and SiHa and normal dermal fibroblast $\mathrm{Hs} 60$. Cells were treated with sesamin at 15, 30, 75, 150, or $300 \mu \mathrm{M}$ for $24 \mathrm{~h}$ (upper panel) or $48 \mathrm{~h}$ (lower panel), and then the cell proliferation was determined using CCK-8 assay. Quantitative data were presented as mean \pm SD. Three independent experiments were performed for statistical analysis. *, **, and ***, $P<0.05,0.01$, and 0.005 as compared to DMSO control. 
apoptotic cells, to $21.3 \pm 3.4 \%$ and $36.5 \pm 4.5 \%$ in HeLa and SiHa cells, respectively $(P<0.005$ as compared to DMSO control). Taken together, these findings suggest that sesamin induces G0/G1 arrest and eventually results in the apoptosis of cervical cancer cells.

\section{Sesamin induces a p53-mediated apoptotic cascade in $\mathrm{SiHa}$ cells}

Based on the observation that remarkable sub-G1 accumulation was clearly induced in SiHa cells in response to sesamin, the effects of sesamin on the apoptotic cascade in $\mathrm{SiHa}$ cells were further investigated. As shown in Figure 3A, sesamin clearly increased p53 protein levels and induced p53 phosphorylation at serine 15 [p-p53(S15)] and serine
46 [p-p53(S46)], in a dose-dependent manner. In addition, PUMA and Bax, the important effectors involved in p53-mediated apoptosis, were also upregulated by sesamin.

PTEN, a tumor suppressor that inhibits AKT activation, can be induced by phosphorylated p53(serine 46). Therefore, whether sesamin induces PTEN expression and subsequently inhibits AKT activation were explored. As shown in Figure 3B, sesamin dose-dependently upregulated PTEN expression, while reducing AKT phosphorylation at serine 473 [p-AKT(S473)] in SiHa cells. Collectively, these results reveal that sesamin upregulates p53 levels and induces p53 activation and the subsequent p53-mediated apoptotic cascade.
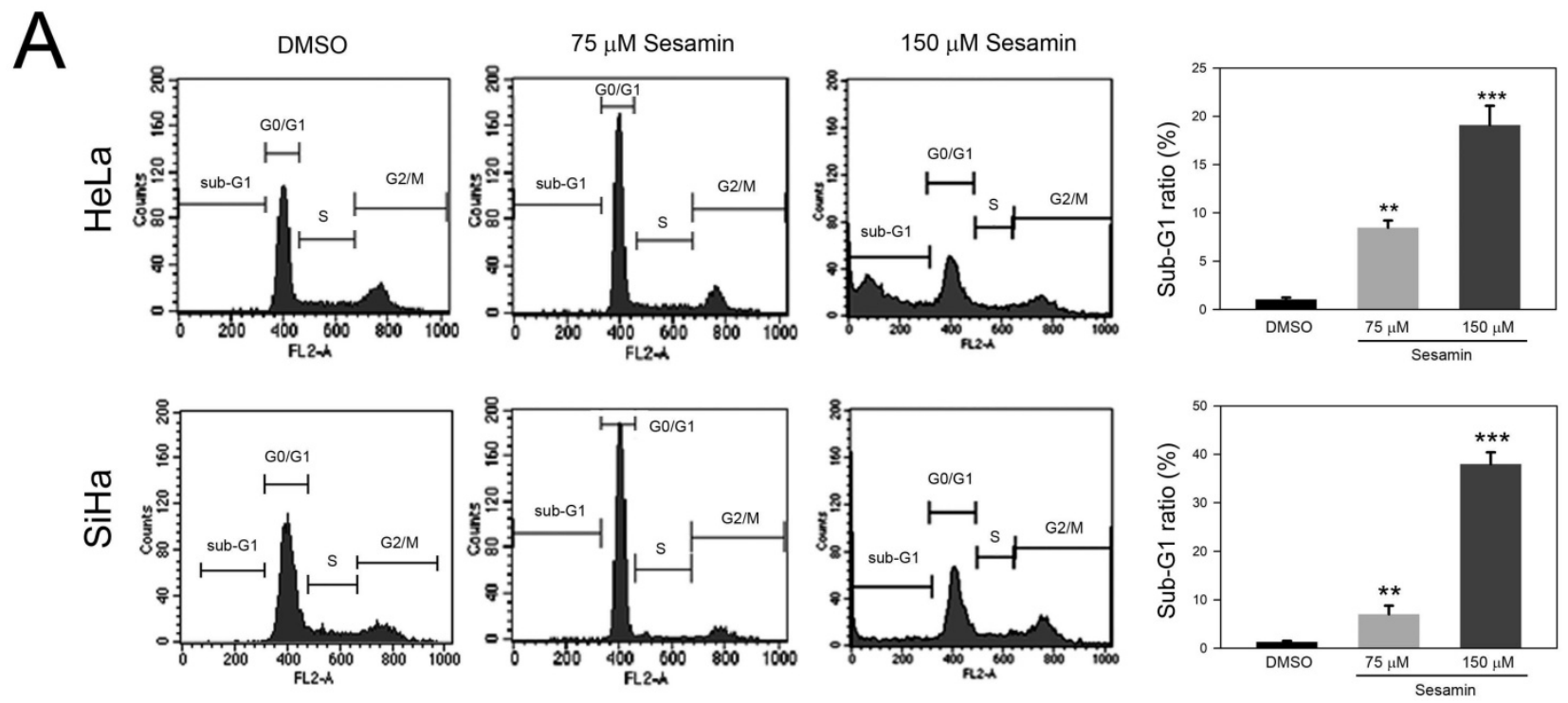

B
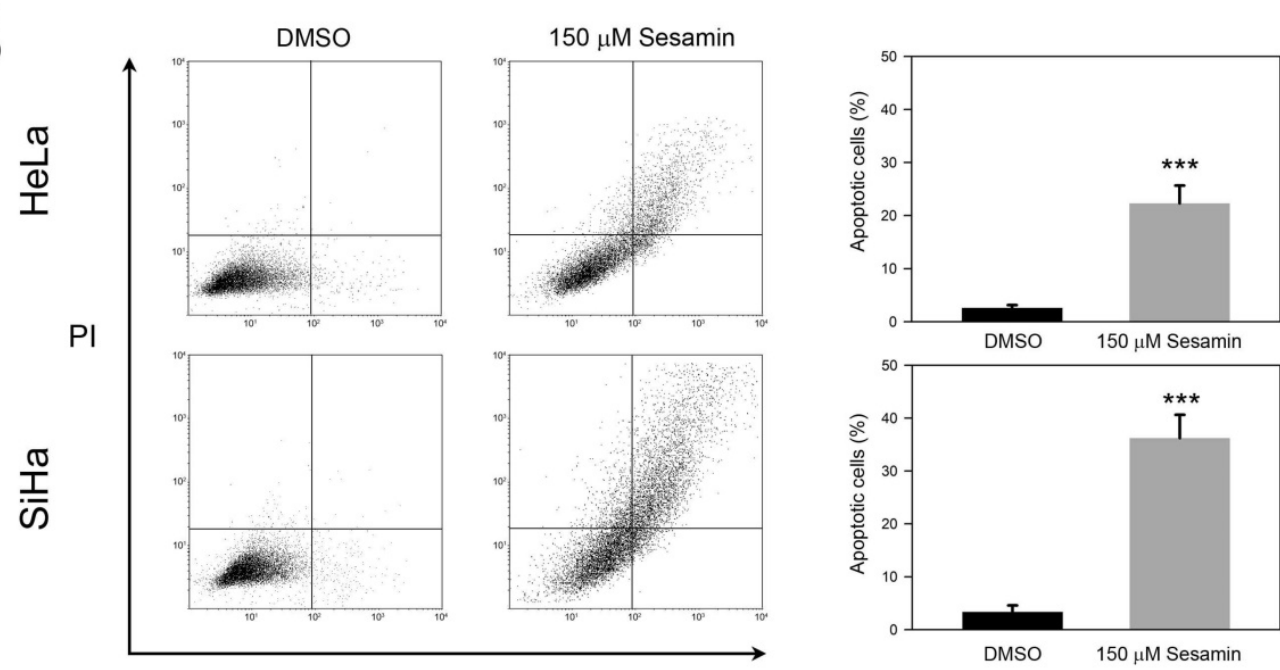

Annexin V

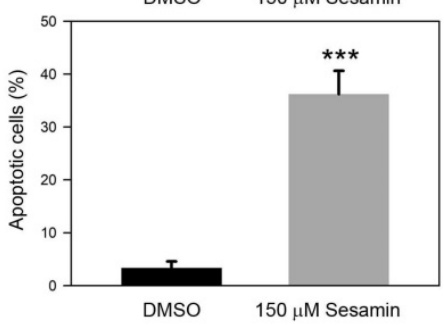

Figure 2. Sesamin interfered cell cycle progression and induced apoptosis of human cervical cancer cell HeLa and SiHa. Cells were treated with sesamin at 75 or $150 \mu \mathrm{M}$ for $24 \mathrm{~h}$, followed by staining with (A) PI for cell cycle distribution, or (B) PI/Annexin V for cell apoptosis assay, and then analyzed using flow cytometry. Quantitative data were presented as mean \pm SD. Three independent experiments were performed for statistical analysis. $* *$, and $* * *, P<0.01$, and 0.005 as compared to DMSO control. 

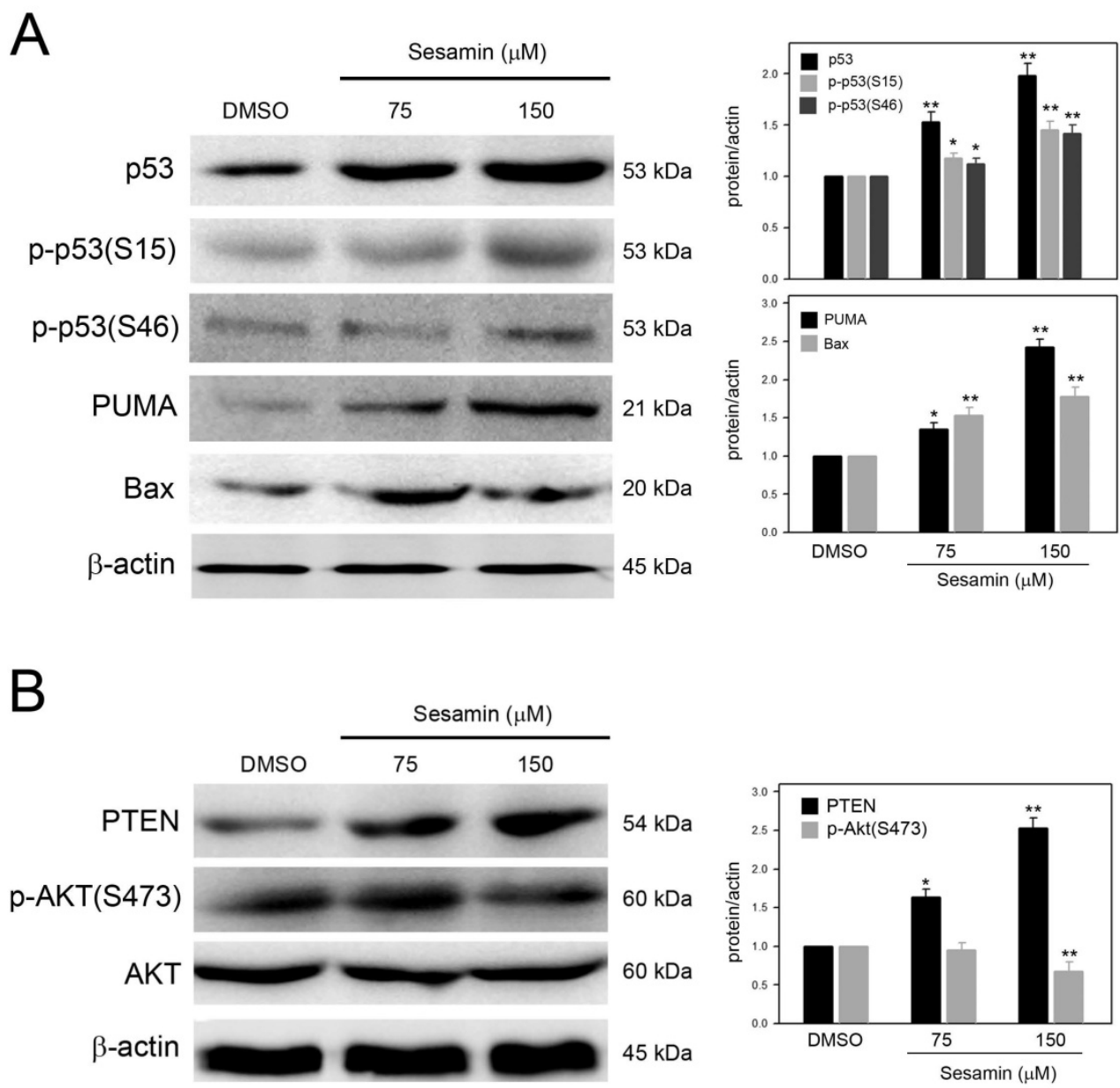

Figure 3. Sesamin induced p53 activation, upregulated expression of PUMA, Bax, and PTEN, and inhibited AKT activation in SiHa cells. Cells were treated with sesamin at 75 or $150 \mu \mathrm{M}$ for $24 \mathrm{~h}$, collected, and then lysed for protein extraction and the subsequent Western blotting. Protein and phosphorylation level were detected using specific antibodies against (A) p53, phosphorylated p53, PUMA, and Bax; and (B) PTEN, AKT, and phosphorylated AKT. Chemiluminescence signal of $\beta$-actin was used as internal control. Molecular weights of signals were indicated. Three independent experiments were performed for statistical analysis. *, and $* *, P<0.05$, and 0.01 as compared to DMSO control.

\section{Involvement of p53 in the apoptotic cascade and proliferation inhibition of cervical cancer cells in response to sesamin}

Based on the observation that sesamin induces a p53-mediated apoptotic cascade and PTEN expression, the involvement of p53 activation in the apoptotic cascade and proliferation inhibition of cervical cancer cells in response to sesamin were next assessed. As shown in Figure 4A, sesamin-induced p53 activation, p-p53(S15) and p-p53(S46), was clearly inhibited by pifithrin- $\alpha$ pretreatment. In addition, pifithrin- $\alpha$ pretreatment also reduced the sesamin-induced expression of PTEN, PUMA, and Bax, but restored AKT activation [p-AKT(S473)] in $\mathrm{SiHa}$ cells. In parallel, pifithrin- $\alpha$ pretreatment also reduced the apoptosis of HeLa and $\mathrm{SiHa}$ cells in response to sesamin (Figure $4 \mathrm{~B}, \mathrm{P}<0.005$ as compared to sesamin alone). Moreover, pifithrin- $\alpha$ pretreatment restored the proliferation of HeLa and SiHa cells in the presence of sesamin (Figure $4 \mathrm{C}, P<0.05$ compared to sesamin alone). Taken together, these findings show that p53 activation is involved in the sesamin-induced apoptotic cascade, as well as in the sesamin-restrained proliferation of $\mathrm{HeLa}$ and $\mathrm{SiHa}$ cells.

\section{Discussion}

Epidemiological studies have shown an inverse relationship between the intake of botanical foods and the incidence of several cancers [11-13]. Consuming dietary plants benefits cancer prevention, in addition to supplementary treatment with phytochemicals that possess various biological activities, including apoptosis induction, cell cycle regulation, tumor suppressor gene activation, oncogenes inactivation, the suppression of tumor proliferation and progression, and the inhibition of angiogenesis, tumorigenesis, and metastasis. Dou et al. reported that sesamin can lead to apoptosis and inhibit the migratory potential of HeLa cells by inducing ER stress and autophagy [14]. Similarly, our results show 

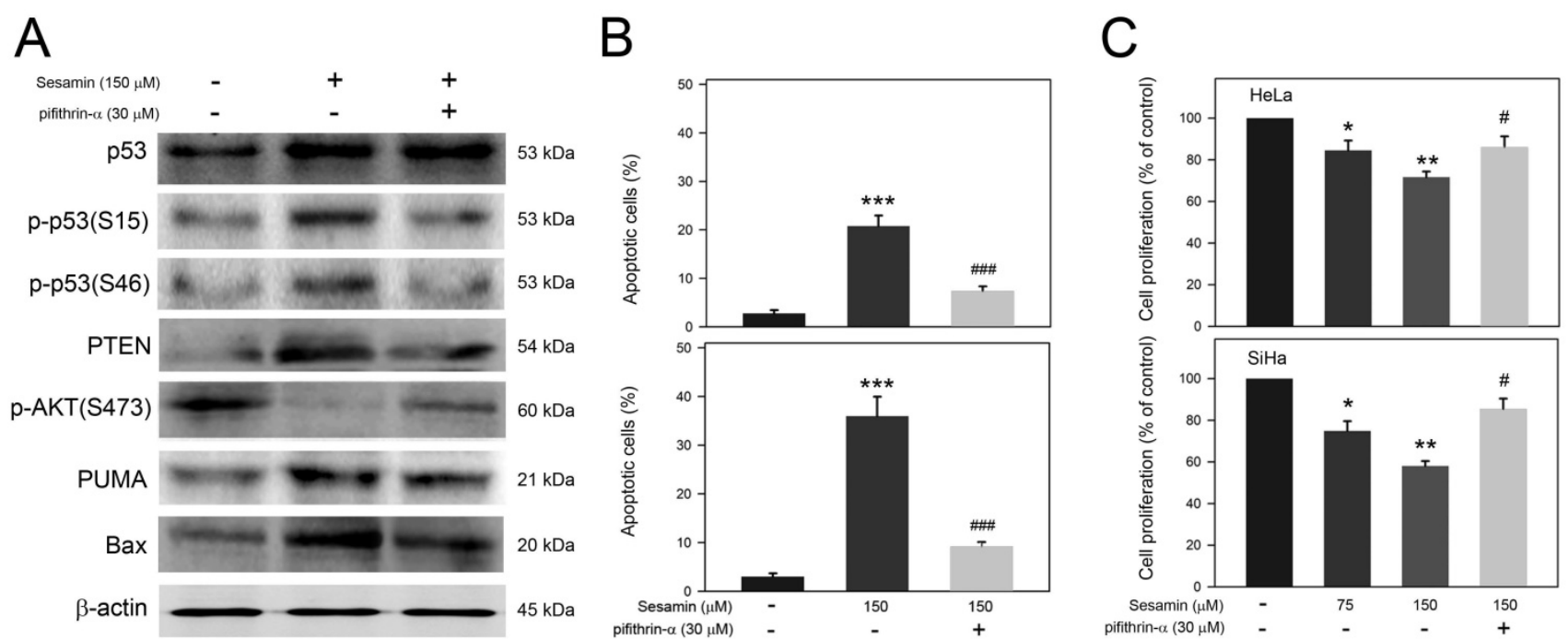

Figure 4. Involvement of p53 activation in sesamin-induced apoptotic cascade in SiHa cells and sesamin-induced cell apoptosis of HeLa and SiHa cells. Cells were pretreated without or with pifithrin-a at $30 \mu \mathrm{M}$ for $2 \mathrm{~h}$, and then treated with sesamin at $150 \mu \mathrm{M}$ for $24 \mathrm{~h}$. The treated cells were collected, and then (A) lysed for protein extraction and the subsequent Western blotting using specific antibodies as indicated; (B) stained with PI/Annexin V for apoptosis assay; and (C) reacted with CCK-8 for cell proliferation assay. Chemiluminescence signal of $\beta$-actin was used as internal control. Molecular weights of signals were indicated. Quantitative data were presented as mean \pm SD. Three independent experiments were performed for statistical analysis. *, ${ }^{* *}$, and $* * *, P<0.05,0.01$, and 0.005 as compared to DMSO control. \# and \#\#, $P<0.05$ and 0.005 as compared to sesamin alone.

that sesamin can disrupt cell cycle progression and inhibit the proliferation of human HeLa and $\mathrm{SiHa}$ cervical cancer cells by inducing p53 activation, which indicate that sesamin may have potential anticancer activity against human cervical cancer.

p53 is a central tumor suppressor that regulates key genes involved in cell cycle regulation, DNA repair, cell survival, and cell death [15]. The p53 cascade governs cell cycle progression by transactivating downstream effectors, such as p21 and p27 [16]. Moreover, activated p53 also upregulates the expression of PTEN [17], and subsequent formation of the PTEN/p53 complex promotes p53 transcriptional activity, thereby further upregulating the expression of PTEN and p21 and leading to cell cycle arrest [18]. In addition, previous studies have reported that sesamin can suppress hepatocellular carcinoma cells via p53/p21-associated STAT3 signaling [19] and non-small cell lung cancer cells via the AKT-mediated p53 pathway [20]. Our findings show that sesamin induced G0/G1 arrest at $75 \mu \mathrm{M}$ and led to remarkable sub-G1 accumulation at $150 \mu \mathrm{M}$. In parallel, p53-induced PTEN also suppresses AKT activation. Collectively, these observations reveal that sesamin may induce cell cycle arrest and further apoptotic cascades by upregulating PTEN expression and consequently inhibiting AKT survival signals.

It is estimated that approximately $99.7 \%$ of cervical cancers carry integrated HPV DNA in the host genome [21]. During HPV infection, two major HPV oncoproteins, E6 and E7, may induce transformation and promote tumorigenesis by binding to $\mathrm{p} 53$ and $\mathrm{pRb}$, thereby enhancing the ubiquitin-dependent proteasomal degradation of p53 and dysregulation of cell cycle progression [22, 23]. To fully abolish p53 activity, E6 also promotes degradation of the p53 downstream apoptosis inducer Bax [24] and inhibits FADD and pro-caspase 8 [25-27]. Our findings reveal that sesamin not only activates p53 by phosphorylating serine 15 and serine 46, but also upregulates the levels of PUMA and Bax, which suggests that sesamin might inhibit the binding of E6 to Bax, thereby reducing Bax degradation. In addition, these findings also indicate that the p53-mediated PUMA/Bax axis plays an important role in the anti-proliferative effect of sesamin on cervical cancer cells.

\section{Conclusions}

In conclusion, our results reveal that sesamin possesses anti-proliferative effects on cervical cancer cells, which may mainly be attributed to p53 activation and p53-mediated apoptosis. These findings not only indicate that sesamin could be a dietary-derived compound with potential anti-proliferative activity against cervical cancer cells, but also reveal that p53/Bax might be a potential target for the development of new cervical cancer treatment.

\section{Funding}

This work was supported by the grants from Chung Shan Medical University (CSMU-CMMC107-04), Chi Mei Medical Center (CMCSMU10705), and from the Ministry of Science and Technology of Taiwan (MOST 108-2320-B-040-026-MY3). 


\section{Competing Interests}

The authors have declared that no competing interest exists.

\section{References}

1. Forouzanfar MH, Foreman KJ, Delossantos AM, Lozano R, Lopez AD, Murray CJ, et al. Breast and cervical cancer in 187 countries between 1980 and 2010: a systematic analysis. Lancet. 2011; 378: 1461-84.

2. Suh DH, Kim M, Kim HJ, Lee KH, Kim JW. Major clinical research advances in gynecologic cancer in 2015. Journal of gynecologic oncology. 2016; 27 : e53.

3. Tangjitgamol S, Levenback CF, Beller U, Kavanagh JJ. Role of surgical resection for lung, liver, and central nervous system metastases in patients with gynecological cancer: a literature review. International journal of gynecological cancer : official journal of the International Gynecological Cancer Society. 2004; 14: 399-422.

4. Kanthan R, Senger JL, Diudea D. Pulmonary lymphangitic carcinomatosis from squamous cell carcinoma of the cervix. World journal of surgical oncology. 2010; 8: 107

5. Park JY, Lim MC, Lim SY, Bae JM, Yoo CW, Seo SS, et al. Port-site and liver metastases after laparoscopic pelvic and para-aortic lymph node dissection for surgical staging of locally advanced cervical cancer. International journal of gynecological cancer : official journal of the International Gynecological Cancer Society. 2008; 18: 176-80.

6. Robin TP, Amini A, Schefter TE, Behbakht K, Fisher CM. Disparities in standard of care treatment and associated survival decrement in patients with locally advanced cervical cancer. Gynecologic oncology. 2016; 143: 319-25.

7. Suh DH, Kim M, Kim K, Kim HJ, Lee KH, Kim JW. Major clinical research advances in gynecologic cancer in 2016: 10-year special edition. Journal of gynecologic oncology. 2017; 28: e45.

8. Dias DA, Urban S, Roessner U. A historical overview of natural products in drug discovery. Metabolites. 2012; 2: 303-36.

9. Hartwig S, St Guily JL, Dominiak-Felden G, Alemany L, de Sanjose S. Estimation of the overall burden of cancers, precancerous lesions, and genital warts attributable to 9-valent HPV vaccine types in women and men in Europe. Infect Agent Cancer. 2017; 12: 19.

10. Scheffner M. Ubiquitin, E6-AP, and their role in p53 inactivation. Pharmacol Ther. 1998; 78: 129-39.

11. Millen AE, Subar AF, Graubard BI, Peters U, Hayes RB, Weissfeld JL, et al. Fruit and vegetable intake and prevalence of colorectal adenoma in a cancer screening trial. The American journal of clinical nutrition. 2007; 86: 1754-64.

12. Li LY, Luo Y, Lu MD, Xu XW, Lin HD, Zheng ZQ. Cruciferous vegetable consumption and the risk of pancreatic cancer: a meta-analysis. World journal of surgical oncology. 2015; 13: 44.

13. Feskanich D, Ziegler RG, Michaud DS, Giovannucci EL, Speizer FE, Willett WC, et al. Prospective study of fruit and vegetable consumption and risk of lung cancer among men and women. Journal of the National Cancer Institute. 2000; 92: 1812-23.

14. Dou H, Yang S, Hu Y, Xu D, Liu L, Li X. Sesamin induces ER stress-mediated apoptosis and activates autophagy in cervical cancer cells. Life Sci. 2018; 200: 87-93.

15. Sionov RV, Haupt Y. The cellular response to p53: the decision between life and death. Oncogene. 1999; 18: 6145-57.

16. Agarwal ML, Agarwal A, Taylor WR, Stark GR. p53 controls both the G2/M and the G1 cell cycle checkpoints and mediates reversible growth arrest in human fibroblasts. Proceedings of the National Academy of Sciences of the United States of America. 1995; 92: 8493-7.

17. Stambolic V, MacPherson D, Sas D, Lin Y, Snow B, Jang Y, et al. Regulation of PTEN transcription by p53. Mol Cell. 2001; 8: 317-25.

18. Brandmaier A, Hou SQ, Shen WH. Cell Cycle Control by PTEN. J Mol Biol. 2017; 429: 2265-77.

19. Deng P, Wang C, Chen L, Wang C, Du Y, Yan X, et al. Sesamin induces cell cycle arrest and apoptosis through the inhibition of signal transducer and activator of transcription 3 signalling in human hepatocellular carcinoma cell line HepG2. Biological \& pharmaceutical bulletin. 2013; 36: 1540-8.

20. Chen Y, Li H, Zhang W, Qi W, Lu C, Huang H, et al. Sesamin suppresses NSCLC cell proliferation and induces apoptosis via Akt/p53 pathway. Toxicol Appl Pharmacol. 2020; 387: 114848.

21. Walboomers JM, Jacobs MV, Manos MM, Bosch FX, Kummer JA, Shah KV, et al. Human papillomavirus is a necessary cause of invasive cervical cancer worldwide. J Pathol. 1999; 189: 12-9.

22. Narisawa-Saito M, Kiyono T. Basic mechanisms of high-risk human papillomavirus-induced carcinogenesis: roles of E6 and E7 proteins. Cancer Sci. 2007; 98: 1505-11.

23. Heck DV, Yee CL, Howley PM, Munger K. Efficiency of binding the retinoblastoma protein correlates with the transforming capacity of the E7 oncoproteins of the human papillomaviruses. Proceedings of the National Academy of Sciences of the United States of America. 1992; 89: 4442-6.

24. Vogt M, Butz K, Dymalla S, Semzow J, Hoppe-Seyler F. Inhibition of Bax activity is crucial for the antiapoptotic function of the human papillomavirus E6 oncoprotein. Oncogene. 2006; 25: 4009-15.
25. Thomas M, Banks L. Human papillomavirus (HPV) E6 interactions with Bak are conserved amongst E6 proteins from high and low risk HPV types. J Gen Virol. 1999; 80 ( Pt 6): 1513-7.

26. Garnett TO, Filippova M, Duerksen-Hughes PJ. Accelerated degradation of FADD and procaspase 8 in cells expressing human papilloma virus 16 E6 impairs TRAIL-mediated apoptosis. Cell Death Differ. 2006; 13: 1915-26.

27. Filippova M, Johnson MM, Bautista M, Filippov V, Fodor N, Tungteakkhun SS, et al. The large and small isoforms of human papillomavirus type 16 E6 bind to and differentially affect procaspase 8 stability and activity. J Virol. 2007; 81: 4116-29. 